\title{
Persistence probabilities of two-sided (integrated) sums of correlated stationary Gaussian sequences
}

\author{
Frank Aurzada Micha Buck
}

September 27, 2018

\begin{abstract}
We study the persistence probability for some two-sided discretetime Gaussian sequences that are discrete-time analogs of fractional Brownian motion and integrated fractional Brownian motion, respectively. Our results extend the corresponding ones in continuous-time in [11] and 12] to a wide class of discrete-time processes.
\end{abstract}

\section{Introduction}

Persistence concerns the probability that a stochastic process has a long negative excursion. In this paper, we are concerned mainly with two-sided discrete-time processes: If $Z=\left(Z_{n}\right)_{n \in \mathbb{Z}}$ is a stochastic process, we study the rate of decay of the probability

$$
\mathbb{P}\left(Z_{n} \leq 0:|n| \leq N\right), \quad \text { as } \quad N \rightarrow \infty .
$$

In many cases of interest, the above probability decreases polynomially, i.e., as $N^{-\theta+o(1)}$, and it is the first goal to find the persistence exponent $\theta$. For a recent overview on this subject, we refer to the surveys [9], [7], [5].

The purpose of this paper is to analyse the persistence probability for the discrete-time analogs of two-sided fractional Brownian motion (FBM) and two-sided integrated fractional Brownian motion (IFBM). Our study extends results in [11] and [12, respectively, to a wide class of discrete-time processes.

The study of persistence probabilities of FBM, IFBM and related processes has received considerable attention in theoretical physics and mathematics, recently. For instance, see [13] and [12] where a relation between the Hausdorff dimension of Lagrangian regular points for the inviscid Burgers equation with FBM initial velocity and the persistence probabilities of IFBM is established; the interest for it arises from [18] and [19]. Further, in [14 a physical model involving FBM is studied as an extension to the Sinai model; see also [2]. Here, persistence probabilities are related to scaling 
properties of a quantity, called steady-state current. Moreover, persistence of non-Markovian processes that are similar to FBM are studied in [8] and [4], confirming results in [16] and [10].

Let us recall that a FBM $\left(W_{H}(t)\right)_{t \in \mathbb{R}}$ is a centered Gaussian process with covariance

$$
\mathbb{E}\left[W_{H}(t) W_{H}(s)\right]=\frac{1}{2}\left(|t|^{2 H}+|s|^{2 H}-|t-s|^{2 H}\right), \quad t, s \in \mathbb{R},
$$

where $0<H<1$ is a constant parameter, called Hurst parameter. For $H=1 / 2$ this is a usual two-sided Brownian motion. For any $0<H<1$, the process has stationary increments, but no independent increments (unless $H=1 / 2)$. Furthermore, it is an $H$-self-similar process. An $\operatorname{IFBM}\left(I_{H}(t)\right)_{t \in \mathbb{R}}$ is defined by $I_{H}(t):=\int_{0}^{t} W_{H}(s) \mathrm{d} s$ and is an $(H+1)$-self-similar process.

In order to define the discrete-time analogs, let $\left(\xi_{n}\right)$ be a real valued stationary centered Gaussian sequence such that

$$
\sum_{j=1}^{n} \sum_{k=1}^{n} \mathbb{E} \xi_{j} \xi_{k} \sim n^{2 H} \ell(n), \quad n \rightarrow \infty,
$$

with $0<H<1$ and $\ell$ slowly varying at infinity. Here and below, we write $f(x) \sim g(x)\left(x \rightarrow x_{0}\right)$ if $\lim f(x) / g(x)=1$ as $x \rightarrow x_{0}$. Then, (1) implies the weak convergence result

$$
\left(\frac{1}{n^{H} \ell(n)^{1 / 2}} \sum_{k=1}^{\lfloor n t\rfloor} \xi_{k}\right)_{t \geq 0} \Rightarrow\left(W_{H}(t)\right)_{t \geq 0}
$$

with fractional Brownian motion $\left(W_{H}(t)\right)$, see e.g. Theorem 4.6.1 in [20]. For this reason, it is natural to consider the stationary increments sequence $\left(S_{n}\right)_{n \in \mathbb{Z}}$ given by

$$
S_{n}-S_{n-1}:=\xi_{n} \text { for } n \in \mathbb{Z} \text { and } S_{0}:=0
$$

as a discrete-time analog of FBM.

Now, we will define the discrete-time analog of IFBM such that symmetry properties like in the continuous-time setting are satisfied. With this in mind, a natural process is given by

$$
I_{n}-I_{n-1}:=\left(S_{n}+S_{n-1}\right) / 2 \text { for } n \in \mathbb{Z} \quad \text { and } \quad I_{0}:=0 .
$$

In Section 2, we discuss relations to the process with increments $\left(S_{n}\right)$ (instead of $\left.\left(\left(S_{n}+S_{n-1}\right) / 2\right)\right)$, which may also seem natural but for which our method of proof does not apply directly due to a lack of symmetry.

In [11] it is shown that one has $\mathbb{P}\left(W_{H}(t) \leq 1:|t| \leq T\right)=T^{-1+o(1)}$. Our first result, treats the discrete-time analog. The technique we use to prove the theorem is completely different from the one in [11]. 
Theorem 1. Let $\left(\xi_{n}\right)$ be a real valued stationary centered Gaussian sequence such that (1) holds. Then, there is a constant $c>0$ such that, for every $N \geq 1$,

$$
c^{-1} N^{-1} \leq \mathbb{P}\left(S_{n} \leq 0 \quad:|n| \leq N\right) \leq N^{-1} .
$$

In order to prove the corresponding result for the process $\left(I_{n}\right)$, we will use a change of measure argument. This argument requires an additional assumption as follows: Let $\mu$ denote the spectral measure of the sequence $\left(\xi_{n}\right)$, i.e.,

$$
\mathbb{E} \xi_{j} \xi_{k}=: \int_{(-\pi, \pi]} e^{i|j-k| u} \mathrm{~d} \mu(u) .
$$

The spectral measure $\mu$ has a (possibly vanishing) component that is absolutely continuous with respect to the Lebesgue measure. Let us denote by $p$ its density, i.e., $\mathrm{d} \mu(u)=: p(u) \mathrm{d} u+\mathrm{d} \mu_{s}(u)$. We will assume that $p$ satisfies

$$
p(u) \sim \ell(1 / u)|u|^{1-2 H}, \quad u \rightarrow 0,
$$

where $\ell$ is a slowly varying function at infinity. It is well-known that (3) implies (11) and thus (2).

The nature of this assumption can be understood by considering the fractional Gaussian noise process, defined by $\xi_{n}^{\mathrm{FGN}}:=W_{H}(n)-W_{H}(n-1)$. This stationary centered Gaussian sequence has an absolutely continuous spectral measure with density function $p_{\mathrm{FGN}}$ that satisfies (see e.g. [17])

$$
p_{\mathrm{FGN}}(u) \sim m_{H}|u|^{1-2 H}, \quad u \rightarrow 0,
$$

where $m_{H}=\Gamma(2 H+1) \sin (\pi H) / 2 \pi$. So, we assume that the density of the absolutely continuous part of the spectral measure of the stationary process $\left(\xi_{n}\right)$ is comparable to the spectral density of fractional Gaussian noise, up to the slowly varying function $\ell$.

We are now ready to state our second main result.

Theorem 2. Let $\left(\xi_{n}\right)$ be a real valued stationary centered Gaussian sequence such that (3) holds. Then,

$$
\mathbb{P}\left(I_{n} \leq 0:|n| \leq N\right)=N^{-(1-H)+o(1)} .
$$

We recall that [12] considers the continuous-time case. Many arguments from that paper can be adapted to our setup. However, for instance, arguments using self-similarity need to be replaced by new ideas. Furthermore, new results concerning the change of measure are needed and may be of independent interest.

For example, as a byproduct of the change of measure techniques, we can improve Theorem 11 in [4], where the persistence problem of the onesided discrete-time analog of FBM is considered. There it is shown that for 
every real valued stationary centered Gaussian sequence $\left(\xi_{n}\right)_{n \in \mathbb{N}}$ such that (11) holds and every $a>0$, there is some constant $c>0$ such that

$$
\begin{aligned}
& c^{-1} N^{-(1-H)} \frac{\sqrt{\ell(N)}}{\sqrt{\log (N)}} \leq \mathbb{P}\left(S_{n}<0: 1 \leq n \leq N\right) \quad \text { and } \\
& \mathbb{P}\left(S_{n}<-a: 1 \leq n \leq N\right) \leq c N^{-(1-H)} \sqrt{\ell(N)} .
\end{aligned}
$$

Thus, one has a lower bound for the probability $\mathbb{P}\left(S_{n}<b: 1 \leq n \leq N\right)$, if $b$ is non-negative, and an upper bound, if $b$ is negative. In order to get both, a lower estimate and an upper estimate, for some arbitrary $b \in \mathbb{R}$, [4] uses a change of measure argument. To get this argument to work, a strong assumption on the covariance function of $\left(S_{n}\right)$ is made; namely $\inf _{n \geq 1} \mathbb{E} S_{1} S_{n}>0$ (see also our Remark 9 below). We are able to prove upper and lower bounds whenever (3) is satisfied. We state this result as Corollary 8 below.

The outline of this paper is as follows. In Section 2, we collect some basic properties of the processes $\left(S_{n}\right)$ and $\left(I_{n}\right)$. Moreover, we present some results concerning the reproducing kernel Hilbert spaces of the considered processes that may be of independent interest. In Section 3, we give a proof of Theorem 1. Finally, in Section 4, we prove our main result, Theorem 2.

\section{Preliminaries}

Let $\left(W_{H}(t)\right)$ be a FBM with Hurst parameter $0<H<1$ and $\left(I_{H}(t)\right)$ an IFBM. Then, unlike $\left(W_{H}(t)\right)$, the process $\left(I_{H}(t)\right)$ does not have stationary increments. Instead, the process satisfies for all $t_{0} \in \mathbb{R}$

$$
\left(I_{H}\left(t+t_{0}\right)-I_{H}\left(t_{0}\right)-t W_{H}\left(t_{0}\right)\right)_{t \in \mathbb{R}} \stackrel{d}{=}\left(I_{H}(t)\right)_{t \in \mathbb{R}} .
$$

In the discrete-time setup, we have analogous properties. From the definition of the process $\left(S_{n}\right)$, we straightforwardly obtain stationary increments

$$
\left(S_{n_{0}+n}-S_{n_{0}}\right)_{n \in \mathbb{Z}} \stackrel{d}{=}\left(S_{n}\right)_{n \in \mathbb{Z}} \quad \text { for all } \quad n_{0} \in \mathbb{Z} .
$$

Also, it is easy to verify that we have

$$
\left(I_{n_{0}+n}-I_{n_{0}}-n \tilde{S}_{n_{0}}\right)_{n \in \mathbb{Z}} \stackrel{d}{=}\left(I_{n}\right)_{n \in \mathbb{Z}} \quad \text { for all } \quad n_{0} \in \mathbb{Z}
$$

where $\left(\tilde{S}_{n}\right)_{n \in \mathbb{Z}}$ denotes the sequence given by $\tilde{S}_{n}:=\frac{S_{n}+S_{n-1}}{2}$.

Let us now recall the definition of the reproducing kernel Hilbert space (RKHS) of a centered Gaussian process $\left(X_{t}\right)_{t \in \mathbb{T}}$. For this purpose, let $\mathbb{H}$ denote the $L^{2}$-closure of the set $\operatorname{span}\left\{X_{t}: t \in \mathbb{T}\right\}$. Then the RKHS $\mathcal{H}$ of $\left(X_{t}\right)$ is the Hilbert space of functions

$$
\mathbb{T} \ni t \mapsto \mathbb{E}\left[X_{t} h\right], \quad h \in \mathbb{H},
$$


with inner product $\left\langle\mathbb{E}\left[X h_{1}\right], \mathbb{E}\left[X h_{2}\right]\right\rangle_{\mathcal{H}}=\mathbb{E}\left[h_{1} h_{2}\right]$.

The following result, Proposition 1.6 in [3], will be an important tool throughout this work.

Proposition 3. Let $X$ be some centered Gaussian process with RKHS $\mathcal{H}$. Denote by $\|\cdot\|$ the norm in $\mathcal{H}$. Then, for each $f \in \mathcal{H}$ and each measurable $S$ such that $\mathbb{P}(X \in S) \in(0,1)$, we have

$$
e^{-\sqrt{2\|f\|^{2} \log (1 / \mathbb{P}(X \in S))}-\|f\|^{2} / 2} \mathbb{P}(X \in S) \leq \mathbb{P}(X+f \in S) .
$$

If $\|f\|^{2}<2 \log (1 / \mathbb{P}(X \in S))$, we have in addition

$$
\mathbb{P}(X+f \in S) \leq e^{\sqrt{2\|f\|^{2} \log (1 / \mathbb{P}(X \in S))}-\|f\|^{2} / 2} \mathbb{P}(X \in S) .
$$

Remark 4. We want to mention that the proof of Proposition 1.6 in 3 fails if $\|f\|^{2} \geq 2 \log (1 / \mathbb{P}(X \in S))$. Thus, unlike in [3], we have excluded this case here. In the applications of this proposition that we know of, the function $f \in \mathcal{H}$ is fixed and one is interested in the asymptotic behavior of the probabilities $\mathbb{P}\left(X \in S^{(N)}\right)$ for $N \rightarrow \infty$, where $\left(S^{(N)}\right)$ is a sequence of measurable sets such that $\lim _{N \rightarrow \infty} \mathbb{P}\left(X \in S^{(N)}\right)=0$. In this case the condition is satisfied for $N$ large enough. Hence, Proposition 1.6 in [3] can be applied in the same way as before.

First, we show the existence of a function in the RKHS of $\left(\xi_{n}\right)_{n \in \mathbb{Z}}$ with certain asymptotic behavior.

Proposition 5. Let $H \in(0,1), \rho \in(-1, H-1)$ and let $\mathcal{H}_{H}(\xi)$ denote the RKHS of the process $\left(\xi_{n}\right)_{n \in \mathbb{Z}}$. Then, if (3) is satisfied, there is an even function $h \in \mathcal{H}_{H}(\xi)$ such that $h>0$ and $h(n) \sim n^{\rho}$.

Proof. Recall that $h \in \mathcal{H}_{H}(\xi)$ if and only if there is a function $\varphi \in L^{2}(\mu)$ with $h(n)=\int_{(-\pi, \pi]} \varphi(u) e^{-i n u} \mathrm{~d} \mu(u)$, see e.g. Comment 2.2 .2 (c) in [1]. In order to prove the Proposition, we will first consider a function $\varphi_{1} \in L^{2}(\mu)$ such that the corresponding function $h_{1} \in \mathcal{H}_{H}(\xi)$ has the correct asymptotic behavior. This function can attain non-positive values at finitely many times. To fix this, we will construct afterwards another function $\varphi_{2} \in L^{2}(\mu)$ such that the corresponding function $h_{2} \in \mathcal{H}_{H}(\xi)$ is non-negative, takes positive values when $h_{1}$ takes non-positive values and decays faster than $h_{1}$. Then, for suitable constants $c_{1}, c_{2}>0$, the function $h=c_{1} h_{1}+c_{2} h_{2}$ has the required properties.

Construction of $h_{1}$ : Due to (3), there is a function $\tilde{\ell}$ and a constant $u_{0}>0$ such that $p(u)=\tilde{\ell}(u)|u|^{1-2 H}$ for $u \in\left[-u_{0}, u_{0}\right]$ and $\tilde{\ell}$ is slowly varying at zero. By Potter's theorem, see Theorem 1.5.6 in [6], $u_{0}$ can be chosen such that $\tilde{\ell}\left(u_{0}\right) / \tilde{\ell}(u) \leq A\left(\frac{|u|}{u_{0}}\right)^{-\delta}$ for $|u|<u_{0}$, fixed $A>1$ and fixed 
$0<\delta<2(H-1-\rho)$. We set

$$
\varphi_{1}(u):= \begin{cases}|u|^{2 H-2-\rho} / \tilde{\ell}(u), & u \in\left[-u_{0}, u_{0}\right] \cap \operatorname{supp}\left(\mu_{s}\right)^{C}, \\ 0, & \text { otherwise. }\end{cases}
$$

Then, $\varphi_{1} \in L^{2}(\mu)$ because

$$
\begin{aligned}
\int_{(-\pi, \pi]}\left|\varphi_{1}(u)\right|^{2} \mathrm{~d} \mu(u) & =\int_{-u_{0}}^{u_{0}} \frac{|u|^{2 H-3-2 \rho}}{\tilde{\ell}(u)} \mathrm{d} u \\
& \leq \frac{A}{\tilde{\ell}\left(u_{0}\right)} \int_{-u_{0}}^{u_{0}}|u|^{2 H-3-2 \rho}\left(\frac{|u|}{u_{0}}\right)^{-\delta} \mathrm{d} u<\infty
\end{aligned}
$$

Here we used that $2 H-3-2 \rho-\delta>-1$. Moreover,

$$
\begin{aligned}
\int_{(-\pi, \pi]} \cos (n u) \varphi_{1}(u) \mathrm{d} \mu(u) & =\int_{-u_{0}}^{u_{0}} \cos (n u)|u|^{-\rho-1} \mathrm{~d} u \\
& =n^{\rho} \int_{-n u_{0}}^{n u_{0}} \cos (v)|v|^{-\rho-1} \mathrm{~d} v \\
& =2 n^{\rho} \int_{0}^{n u_{0}} \cos (v)|v|^{-\rho-1} \mathrm{~d} v .
\end{aligned}
$$

Since $-\rho-1<0$, it is easy to show, using the Leibniz criterion and the concavity of $(\cdot)^{-\rho-1}$, that the latter integral converges to a constant $c / 2>0$. Thus,

$$
h_{1}(n)=\int_{(-\pi, \pi]} \varphi_{1}(u) e^{-i n u} \mathrm{~d} \mu(u) \sim c n^{\rho} .
$$

Construction of $h_{2}$ : Choose $n_{0}$ such that $h_{1}$ attains only positive values for $|n|>n_{0}$. Let $g \in C^{1}$ be an even real-valued function with support contained in $\left[-u_{0} / 2, u_{0} / 2\right]$ such that the Fourier coefficients for $|n| \leq n_{0}$ do not vanish, e.g. take any smooth even function $g$ with $g(u)>0$ for $|u|<\min \left(u_{0} / 2, \pi /\left(2 n_{0}\right)\right)$ and $g(u)=0$ otherwise. Then, the function $f$ given by $f(u):=\frac{1}{2 \pi} \int_{-\pi}^{\pi} g(v) \bar{g}(u-v) \mathrm{d} v$ has Fourier coefficients $\hat{f}_{n}=\left|\hat{g}_{n}\right|^{2}$. In particular $\hat{f}_{n}>0$ for $|n| \leq n_{0}$. Moreover, $f \in C^{2}$ because $f$ is a convolution of two differentiable functions. Thus, we have

$$
0 \leq \hat{f}_{n}=\frac{1}{(i n)^{2}}\left(\widehat{f^{\prime \prime}}\right)_{n} \leq \frac{\sup _{x \in(-\pi, \pi]}\left|f^{\prime \prime}(x)\right|}{|n|^{2}} \text { for } n \in \mathbb{Z} \backslash\{0\} .
$$

Now, we consider the function

$$
\varphi_{2}(u):= \begin{cases}\frac{f(u)}{|u|^{1-2 H \tilde{\ell}(u)}}, & u \in\left[-u_{0}, u_{0}\right] \cap \operatorname{supp}\left(\mu_{s}\right)^{C}, \\ 0, & \text { otherwise. }\end{cases}
$$


Let $M$ denote the maximum of $f$, then

$$
\begin{aligned}
\int_{(-\pi, \pi]}\left|\varphi_{2}(u)\right|^{2} \mathrm{~d} \mu(u) & \leq \int_{-u_{0}}^{u_{0}} \frac{M^{2}}{|u|^{1-2 H \tilde{\ell}(u)}} \mathrm{d} u \\
& \leq \frac{A}{\tilde{\ell}\left(u_{0}\right)} \int_{-u_{0}}^{u_{0}} \frac{M^{2}}{|u|^{1-2 H}}\left(\frac{|u|}{u_{0}}\right)^{-\delta} \mathrm{d} u<\infty
\end{aligned}
$$

since $2 H-1-\delta>-1$. Furthermore, we have by construction of $\varphi_{2}$

$$
h_{2}(n)=\int_{(-\pi, \pi]} \varphi_{2}(u) e^{-i n u} \mathrm{~d} \mu(u)=\int_{-\pi}^{\pi} f(u) e^{-i n u} \mathrm{~d} u=\hat{f}_{n} .
$$

As a corollary of Proposition 5, we show the existence of functions with certain asymptotic behavior in the RKHSs of $\left(S_{n}\right)_{n \in \mathbb{Z}}$ and $\left(I_{n}\right)_{n \in \mathbb{Z}}$, respectively.

Corollary 6. Let $H \in(0,1), \rho \in(-1, H-1)$ and let $\mathcal{H}_{H}(S)$ and $\mathcal{H}_{H}(I)$ denote the RKHS of the processes $\left(S_{n}\right)_{n \in \mathbb{Z}}$ and $\left(I_{n}\right)_{n \in \mathbb{Z}}$, respectively. Then, if (3) is satisfied, there are functions $f \in \mathcal{H}_{H}(S), g \in \mathcal{H}_{H}(I)$ such that $f$ is odd with $f(n)>0$ for $n>0$ and $f(n) \sim n^{\rho+1}$ as $n \rightarrow \infty$ whereas $g$ is even and positive on $\mathbb{Z} \backslash\{0\}$ with $g(n) \sim n^{\rho+2}$ as $n \rightarrow \infty$.

Proof. Let $h \in \mathcal{H}_{H}(\xi)$ be the positive and even function in Proposition 5 with $h(n) \sim n^{\rho}$. Then, by the definition of the RKHS, there is a random variable $X$ in the $L^{2}$-closure of the set $\operatorname{span}\left\{\xi_{n}: n \in \mathbb{Z}\right\}$ with $h(n)=$ $\mathbb{E}\left[\xi_{n} X\right]$. Now, let the functions $f, g$ be given by $f(n)=(\rho+1) \mathbb{E}\left[S_{n} X\right]$ and $g(n)=(\rho+1)(\rho+2) \mathbb{E}\left[I_{n} X\right]$, respectively. Since the sets $\operatorname{span}\left\{\xi_{n}: n \in \mathbb{Z}\right\}$, $\operatorname{span}\left\{S_{n}: n \in \mathbb{Z}\right\}$ and $\operatorname{span}\left\{I_{n}: n \in \mathbb{Z}\right\}$ coincide, we have $f \in \mathcal{H}_{H}(S)$ and $g \in \mathcal{H}_{H}(I)$. By $h(n) \sim n^{\rho}$ and the symmetry of $h$, we have $-f(-n)=$ $f(n)=(\rho+1) \sum_{k=1}^{n} h(k) \sim n^{\rho+1}$ as $n \rightarrow \infty$. Thus, we have further $g(-n)=$ $g(n)=(\rho+1)(\rho+2) \sum_{k=1}^{n-1} \mathbb{E}\left[S_{k} X\right]+\mathbb{E}\left[S_{n} X\right] / 2 \sim n^{\rho+2}$ as $n \rightarrow \infty$.

As a first application of Corollary 6, we compare the persistence probabilities of $\left(I_{n}\right)$ to a closely related process. Let $\left(\bar{I}_{n}\right)_{n \in \mathbb{Z}}$ be the sequence given by $\bar{I}_{n}-\bar{I}_{n-1}:=S_{n}$ for $n \in \mathbb{Z}$ and $\bar{I}_{0}:=0$. This process is related to the process $\left(I_{n}\right)$ by the identity $\bar{I}_{n}=I_{n}+S_{n} / 2$. Both processes are defined as integrals of stationary increments sequences that have FBM as scaling limit. In the context of this paper, the major difference between these processes is that $\left(I_{n}\right)$ vanishes only at 0 and satisfies $\left(I_{n}\right) \stackrel{d}{=}\left(I_{-n}\right)$ whereas $\bar{I}_{-1}=\bar{I}_{0}=0$ and $\bar{I}_{1}$ does not vanish. The symmetry property of $\left(I_{n}\right)$ resembles the continuoustime case and is needed in the proof of Theorem 2. In the following corollary, we relate the persistence probabilities of both processes. 
Corollary 7. Let $\left(\xi_{n}\right)$ be a real valued stationary centered Gaussian sequence such that (3) holds. Then,

$$
\mathbb{P}\left(\bar{I}_{n} \leq 0:-N-1 \leq n \leq N\right) \leq \mathbb{P}\left(I_{n} \leq 0:|n| \leq N\right) .
$$

If in addition $\mathbb{E}\left[\bar{I}_{n} \bar{I}_{m}\right] \geq 0$ for all $n, m \in \mathbb{Z}$, then one has

$$
\mathbb{P}\left(I_{n} \leq 0:|n| \leq N\right) \leq \mathbb{P}\left(\bar{I}_{n} \leq 0:|n| \leq N\right) \ell_{0}(N),
$$

where $\ell_{0}$ denotes a slowly varying function at infinity.

Proof. The first inequality follows directly from the definitions of the processes, since one has $I_{n}=\left(\bar{I}_{n}+\bar{I}_{n-1}\right) / 2$ for all $n \in \mathbb{Z}$. Using Slepian's Lemma and the additional assumption about the correlations of $\left(\bar{I}_{n}\right)$, we obtain

$$
\begin{aligned}
\mathbb{P}\left(\bar{I}_{n} \leq 0:|n| \leq N\right) \geq & \mathbb{P}\left(\bar{I}_{n} \leq 0:|n| \leq \log (N)\right) \\
& \cdot \mathbb{P}\left(\bar{I}_{n} \leq 0: \log (N)<|n| \leq N\right) .
\end{aligned}
$$

By the same argument and Theorem 1, we have

$$
\begin{aligned}
\mathbb{P}\left(\bar{I}_{n} \leq 0:|n| \leq \log (N)\right) \geq & \mathbb{P}\left(\bar{I}_{n} \leq 0: 0 \leq n \leq \log (N)\right) \\
& \cdot \mathbb{P}\left(\bar{I}_{n} \leq 0:-\log (N) \leq n<0\right) \\
\geq & \mathbb{P}\left(S_{n} \leq 0: 0 \leq n \leq \log (N)\right) \\
& \cdot \mathbb{P}\left(S_{n} \geq 0:-\log (N) \leq n<0\right) \\
\geq & c^{-2} \log (N)^{-2} .
\end{aligned}
$$

Thus, the first factor on the right hand side in (8) can be estimated by a slowly varying function at infinity. It remains to relate the second factor on the right hand side in (8) to the probability $\mathbb{P}\left(I_{n} \leq 0:|n| \leq N\right)$.

By Corollary [6, for $\varepsilon \in(0,1 / 4)$, there is a symmetric function $f \in \mathcal{H}_{H}(I)$ such that $f(n) \geq|n|^{1+H-\varepsilon}$ for all $n \in \mathbb{Z}$. Obviously, we have

$$
\begin{aligned}
\mathbb{P}\left(I_{n} \leq-n^{1+H-\varepsilon}\right. & : \log (N)<|n| \leq N) \\
\leq & \mathbb{P}\left(\bar{I}_{n} \leq 0: \log (N)<|n| \leq N\right) \\
& +\mathbb{P}\left(\exists n: \bar{I}_{n}-I_{n}>n^{1+H-\varepsilon}, \log (N)<|n| \leq N\right) .
\end{aligned}
$$

We will see that the second term on the right hand side is of lower order, while the term on the left hand side can be related to $\mathbb{P}\left(I_{n} \leq 0:|n| \leq N\right)$. For this purpose, let $X$ denote a standard normal random variable. Then, by using $\bar{I}_{n}-I_{n}=S_{n} / 2$ in the first step and (11) in the second step, we have 
for $N$ large enough

$$
\begin{aligned}
\mathbb{P}(\exists n & \left.: \bar{I}_{n}-I_{n}>n^{1+H-\varepsilon}, \log (N)<|n| \leq N\right) \\
& \leq 2 \sum_{n=\lceil\log (N)\rceil}^{N} \mathbb{P}\left(S_{n} / 2>n^{1+H-\varepsilon}\right) \\
& \leq 2 \sum_{n=\lceil\log (N)\rceil}^{N} \mathbb{P}\left(n^{H+\varepsilon} X>n^{1+H-\varepsilon}\right) \\
& \leq 2 N \mathbb{P}\left(X>\log (N)^{1-2 \varepsilon}\right) \\
& \leq 2 N e^{-(\log (N))^{2-4 \varepsilon} / 2} \\
& \leq 2 N^{-2} .
\end{aligned}
$$

In the fourth step above, we used the standard estimate $\mathbb{P}(X>x) \leq e^{-x^{2} / 2}$ for $x \geq 1$. Finally, using Proposition 3, we obtain for $N$ large enough

$$
\begin{aligned}
\mathbb{P}\left(I_{n} \leq 0:|n| \leq N\right) \leq & \mathbb{P}\left(I_{n} \leq 0: \log (N)<|n| \leq N\right) \\
\leq & \mathbb{P}\left(I_{n} \leq-f(n): \log (N)<|n| \leq N\right) \\
& \cdot e^{\sqrt{2\|f\|^{2} \log \left(1 / \mathbb{P}\left(I_{n} \leq 0:|n| \leq N\right)\right)}-\|f\|^{2} / 2} \\
\leq & \mathbb{P}\left(I_{n} \leq-n^{1+H-\varepsilon}: \log (N)<|n| \leq N\right) \\
& \cdot e^{\sqrt{2\|f\|^{2} \log \left(1 / \mathbb{P}\left(I_{n} \leq 0:|n| \leq N\right)\right)}-\|f\|^{2} / 2} .
\end{aligned}
$$

This, together with (9), (10) and Theorem 2, finishes the proof.

As another application of Corollary [6, we can give an improvement of Theorem 11 in [4]:

Corollary 8. Let $\left(\xi_{n}\right)$ be a real valued stationary centered Gaussian sequence such that (3) holds. Then, for every $b \in \mathbb{R}$ there is some constant $c>0$ such that

$$
\begin{aligned}
N^{-(1-H)} \sqrt{\ell(N)} e^{-c \sqrt{\log (N)}} & \leq \mathbb{P}\left(\max _{1 \leq n \leq N} S_{n} \leq b\right) \\
& \leq N^{-(1-H)} \sqrt{\ell(N)} e^{c \sqrt{\log (N)}} \quad \forall N \in \mathbb{N} .
\end{aligned}
$$

Proof. Let $a>0$. By Corollary [6, there is a function $f \in \mathcal{H}_{H}(S)$ with $f(n) \geq 2 a$ for all $n \geq 1$. Further, using the lower estimate in (4), we have for $N$ large enough

$$
N^{-1} \leq \mathbb{P}\left(S_{n} \leq a: 1 \leq n \leq N\right) .
$$


This together with Proposition 3 yields for $N$ large enough

$$
\begin{aligned}
\mathbb{P}\left(S_{n} \leq-a\right. & : 1 \leq n \leq N) \\
& =\mathbb{P}\left(S_{n}+f(n) \leq-a+f(n): 1 \leq n \leq N\right) \\
& \geq \mathbb{P}\left(S_{n}+f(n) \leq a: 1 \leq n \leq N\right) \\
& \geq \mathbb{P}\left(S_{n} \leq a: 1 \leq n \leq N\right) e^{-\sqrt{2\|f\|^{2} \log (N)}-\|f\| / 2} .
\end{aligned}
$$

Combining this with (4) finishes the proof.

Remark 9. In Theorem 11 in [4], the authors assume $\inf _{n \geq 1} \mathbb{E} S_{n} S_{1}>0$ to get the change of measure argument to work. For instance, the fractional Gaussian noise process $\left(\xi_{n}^{\mathrm{FGN}}\right)$ satisfies this assumption. This can be easily verified by using that $\mathbb{E} S_{n}^{2}=n^{2 H}$. In general, this does not remain true if one only has (11). For example, consider the case where $\ell(x)=1+\cos (\pi x) / \log (x)$ in (11). Then, one has $\sum_{j=1}^{n} \sum_{k=1}^{n} \mathbb{E} \xi_{j} \xi_{k} \sim n^{2 H}$ but the function $\mathbb{E} S_{n} S_{1}$ attains infinitely often positive and negative values.

Remark 10. Consider the function $f: \mathbb{N} \rightarrow \mathbb{R}$ with $f(n)=\mathbb{1}_{n=1}$. Clearly, $f$ is in the RKHS of the process $\left(\xi_{n}\right)_{n \geq 1}$ if and only if $\xi_{1} \notin \mathbb{H}_{2}$, where $\mathbb{H}_{2}$ denotes the $L^{2}$-closure of the set $\operatorname{span}\left\{\xi_{n}: n \geq 2\right\}$. It is well known that this condition is equivalent to the Kolmogorov condition

$$
\int_{-\pi}^{\pi} \log (p(u)) \mathrm{d} u>-\infty
$$

where $p$ denotes the density of the component of the spectral measure of $\left(\xi_{n}\right)$ that is absolutely continuous with respect to the Lebesgue measure, see e.g. Theorem 2.5.4 in [1]. In this case, all constant functions are in the RKHS of the process $\left(S_{n}\right)_{n \geq 1}$. Hence, the proof of Corollary 8 still works if we replace condition (3) by (11) and (11).

\section{Proof of Theorem 1}

\section{Upper bound}

Let $T_{N}$ denote the time where the process $\left(S_{n}\right)_{n \in \mathbb{Z}}$ attains its maximum on $\{0,1, \ldots, N\}$. Since $\left(S_{n}\right)_{n \in \mathbb{Z}}$ has stationary increments and $\mathbb{P}\left(S_{j}=S_{k}\right)=0$ 
for $j \neq k$, the upper bound follows from

$$
\begin{aligned}
N \cdot \mathbb{P}\left(S_{n} \leq 0:-N \leq n \leq N\right) & \leq \sum_{k=1}^{N} \mathbb{P}\left(S_{n} \leq 0:-k \leq n \leq N-k\right) \\
& =\sum_{k=1}^{N} \mathbb{P}\left(S_{n} \leq S_{k} \quad: \quad 0 \leq n \leq N\right) \\
& =\sum_{k=1}^{N} \mathbb{P}\left(T_{N}=k\right) \\
& \leq 1 .
\end{aligned}
$$

\section{Lower bound}

Using again the stationary increments of $\left(S_{n}\right)_{n \in \mathbb{Z}}$, we obtain

$$
\begin{aligned}
(N+1) & \cdot \mathbb{P}\left(S_{n} \leq 0:-N \leq n \leq N\right) \\
& \geq \sum_{k=0}^{N} \mathbb{P}\left(S_{n} \leq 0:-N-k \leq n \leq 2 N-k\right) \\
& =\sum_{k=0}^{N} \mathbb{P}\left(S_{n} \leq S_{N+k}: 0 \leq n \leq 3 N\right) \\
& =\sum_{k=0}^{N} \mathbb{P}\left(T_{3 N}=N+k\right) \\
& =\mathbb{P}\left(T_{3 N} \in[N, 2 N]\right) .
\end{aligned}
$$

Now, we consider the continuous functional $F:\left(D([0,1]),\|\cdot\|_{\infty}\right) \rightarrow(\mathbb{R},|\cdot|)$ given by

$$
F(g)=\left(\sup _{x \in\left(\frac{1}{3}, \frac{2}{3}\right)} g(x)-\sup _{x \in\left(0, \frac{1}{3}\right) \cup\left(\frac{2}{3}, 1\right)} g(x)\right)_{+} \wedge 1,
$$

where $(x)_{+}:=\max (x, 0)$ for $x \in \mathbb{R}$ and $D([0,1])$ denotes the set of all càdlàg functions on $[0,1]$. We set

$$
Y_{N}(t)=\frac{1}{N^{H} \ell(N)^{1 / 2}} \sum_{k=1}^{\lfloor N t\rfloor} \xi_{k} .
$$

Due to (2), it follows that

$$
\mathbb{P}\left(T_{3 N} \in[N, 2 N]\right)=\mathbb{E}\left[\mathbb{1}_{T_{3 N} \in[N, 2 N]}\right] \geq \mathbb{E} F\left(Y_{N}\right) \rightarrow c_{0}>0, \quad \text { as } N \rightarrow \infty .
$$

This and (12) show the lower bound. 


\section{Proof of Theorem 2}

The proof is structured as follows: We first consider the functional

$$
F_{N}:=\sum_{k=1}^{N-1}\left(\gamma_{k, k}^{-}-\gamma_{k, N-k}^{+}\right)_{+},
$$

where for $k \in \mathbb{Z}$ and $m \in \mathbb{N}$

$$
\gamma_{k, m}^{-}:=\min _{1 \leq n \leq m} \frac{I_{k}-I_{k-n}}{n} \text { and } \gamma_{k, m}^{+}:=\max _{1 \leq n \leq m} \frac{I_{k+n}-I_{k}}{n},
$$

and determine the polynomial order of $\mathbb{E} F_{N}$ as $N \rightarrow \infty$. Then, we relate the quantity $\mathbb{E} F_{N}$ to the probability

$$
\tilde{p}_{N}:=\mathbb{P}\left(I_{n}+|n| \leq 0,|n| \leq N\right) .
$$

Finally, we obtain the asymptotic order of

$$
p_{N}:=\mathbb{P}\left(I_{n} \leq 0:|n| \leq N\right)
$$

from (13) by using a change of measure argument (Proposition 3 and Corollary 6).

\section{Upper bound for $\mathbb{E} \boldsymbol{F}_{N}$}

In the following, we fix $N$ and write $\gamma_{k}^{-}=\gamma_{k, k}^{-}$and $\gamma_{k}^{+}=\gamma_{k, N-k}^{+}$to ease notation. Let $C_{N}:[0, N] \rightarrow \mathbb{R}$ denote the concave majorant of $I_{n}$ on $[0, N]$, i.e., $C_{N}$ is the smallest concave function with $I_{n} \leq C_{N}(n)$. Obviously, $C_{N}$ is a piecewise linear function and we denote by $\left\{k_{1}, k_{2}, \ldots\right\}$ (depending on $N$ ) its nodal points. At these points the slope on the left is $\gamma_{k_{i}}^{-}$and the slope on the right is $\gamma_{k_{i}}^{+}$. Further, we note that $\gamma_{k}^{-}-\gamma_{k}^{+} \geq 0$ if and only if $k$ is a nodal point of $C_{N}$. In that case one has $\gamma_{k_{i}}^{+}=\gamma_{k_{i+1}}^{-}$. Thus,

$$
F_{N}=\sum_{k=1}^{N-1}\left(\gamma_{k}^{-}-\gamma_{k}^{+}\right)_{+}=\sum_{i}\left(\gamma_{k_{i}}^{-}-\gamma_{k_{i+1}}^{-}\right)=\gamma_{0}^{+}-\gamma_{N}^{-} \text {. }
$$

By $\mathbb{E} \tilde{S}_{N}=0$, (5) and $\left(I_{n}\right) \stackrel{d}{=}\left(I_{-n}\right)$, we have

$$
\begin{aligned}
\mathbb{E}\left[-\gamma_{N}^{-}\right] & =\mathbb{E}\left[-\min _{1 \leq n \leq N} \frac{I_{N}-I_{N-n}}{n}\right] \\
& =\mathbb{E}\left[\max _{1 \leq n \leq N} \frac{I_{N-n}-I_{N}-(-n) \tilde{S}_{N}}{n}\right] \\
& =\mathbb{E}\left[\max _{1 \leq n \leq N} \frac{I_{-n}}{n}\right]=\mathbb{E}\left[\max _{1 \leq n \leq N} \frac{I_{n}}{n}\right]=\mathbb{E} \gamma_{0}^{+} .
\end{aligned}
$$


Therefore,

$$
\mathbb{E} F_{N}=2 \mathbb{E} \gamma_{0}^{+} .
$$

Due to (15), one obtains the upper estimate

$$
\begin{aligned}
\mathbb{E} F_{N} & =2 \mathbb{E}\left[\max _{1 \leq n \leq N} \frac{\sum_{k=1}^{n} \tilde{S}_{k}}{n}\right] \\
& \leq 2 \mathbb{E}\left[\max _{1 \leq n \leq N} \frac{\sum_{k=1}^{n} \max _{1 \leq j \leq N} \tilde{S}_{j}}{n}\right]=2 \mathbb{E}\left[\max _{1 \leq j \leq N} \tilde{S}_{j}\right] .
\end{aligned}
$$

It can be obtained from (2) that

$$
\frac{1}{N^{H} \ell(N)^{1 / 2}} \mathbb{E}\left[\max _{1 \leq n \leq N} \tilde{S}_{n}\right] \rightarrow \mathbb{E}\left[\sup _{t \in[0,1]} W_{H}(t)\right] \in(0, \infty),
$$

where $\left(W_{H}(t)\right)$ is a fractional Brownian motion, see e.g. proof of Theorem 11 in [4]. Thus, there is a constant $c$ such that for all $N$

$$
\mathbb{E} F_{N} \leq c \ell(N)^{1 / 2} N^{H} .
$$

In the following, $c$ will denote a varying positive constant independent of $N$ for ease of notation.

\section{Lower bound for $\mathbb{E} \boldsymbol{F}_{N}$}

Since $\left(\xi_{n}\right)$ is a stationary process, we have

$$
\mathbb{E} S_{j} S_{k}=\frac{1}{2}\left(\mathbb{E} S_{j}^{2}+\mathbb{E} S_{k}^{2}-\mathbb{E} S_{|j-k|}^{2}\right) .
$$

Consequently,

$$
\mathbb{E}\left(I_{N}+\frac{S_{N}}{2}\right)^{2}=\sum_{j=1}^{N} \sum_{k=1}^{N} \mathbb{E} S_{j} S_{k}=\frac{1}{2} \sum_{j=1}^{N} \sum_{k=1}^{N}\left(\mathbb{E} S_{j}^{2}+\mathbb{E} S_{k}^{2}-\mathbb{E} S_{|j-k|}^{2}\right) .
$$

Counting how often $\mathbb{E} S_{k}^{2}$ is added, yields

$$
\begin{aligned}
\mathbb{E}\left(I_{N}+\frac{S_{N}}{2}\right)^{2} & =\frac{1}{2} \sum_{k=1}^{N} N \mathbb{E} S_{k}^{2}+\frac{1}{2} \sum_{k=1}^{N} N \mathbb{E} S_{k}^{2}-\sum_{k=1}^{N}(N-k) \mathbb{E} S_{k}^{2} \\
& =\sum_{k=1}^{N} k \mathbb{E} S_{k}^{2} .
\end{aligned}
$$

Since $\mathbb{E} S_{n}^{2} \sim n^{2 H} \ell(n)$, we can apply Proposition 1.5.8 in [6] to obtain

$$
\mathbb{E}\left(I_{N}+\frac{S_{N}}{2}\right)^{2} \sim N^{2 H+2} \ell(N) /(2 H+2) .
$$


Now, using the Cauchy-Schwarz Inequality, we have $\left|\mathbb{E} S_{N} I_{N}\right| \leq \sqrt{\mathbb{E} S_{N}^{2} \mathbb{E} I_{N}^{2}}$ and we can thus conclude from (17) that

$$
\mathbb{E} I_{N}^{2} \sim N^{2 H+2} \ell(N) /(2 H+2) .
$$

In the following, we let $\|\cdot\|_{2}$ denote the norm $\|X\|_{2}=\mathbb{E}\left[|X|^{2}\right]^{1 / 2}$. Moreover, we recall the identity $\mathbb{E} X_{+}=(2 \pi)^{-1 / 2}\|X\|_{2}$ for a centered normal random variable $X$. Now, we can give a lower bound for $\mathbb{E} F_{N}$. By (15) and $\mathbb{E} I_{1}=0$, we have

$$
\begin{aligned}
\mathbb{E} F_{N} & =2 \mathbb{E}\left[\max _{1 \leq n \leq N} \frac{I_{n}}{n}\right]=2 \mathbb{E}\left[\max _{1 \leq n \leq N} \frac{I_{n}}{n}-I_{1}\right] \\
& =2 \mathbb{E}\left(\max _{1 \leq n \leq N} \frac{I_{n}}{n}-I_{1}\right)_{+} \geq 2 \mathbb{E}\left(\frac{I_{N}}{N}-I_{1}\right)_{+} \\
& =\sqrt{2 / \pi}\left\|\frac{I_{N}}{N}-I_{1}\right\|_{2} \geq \sqrt{2 / \pi}\left(\left\|\frac{I_{N}}{N}\right\|_{2}-\left\|I_{1}\right\|_{2}\right) .
\end{aligned}
$$

Thus, by (18), we have

$$
\mathbb{E} F_{N} \geq c^{-1} \ell(N)^{1 / 2} N^{H}
$$

\section{Upper bound for $\tilde{p}_{N}$}

In order to get an upper bound for the probability in (13), it is convenient to consider the random variable

$$
\vartheta_{N}:=\left(\gamma_{0, N}^{-}-\gamma_{0, N}^{+}\right)_{+} .
$$

We have

$$
\mathbb{E}\left(\gamma_{k}^{-}-\gamma_{k}^{+}\right)_{+} \geq \mathbb{E} \vartheta_{N} .
$$

To see this, note that by using (프), we obtain

$$
\begin{aligned}
\gamma_{k, N}^{-}-\gamma_{k, N}^{+} & =\min _{1 \leq n \leq N}-\frac{I_{k-n}-I_{k}-(-n) \tilde{S}_{k}}{n}-\max _{1 \leq n \leq N} \frac{I_{k+n}-I_{k}-n \tilde{S}_{k}}{n} \\
& \stackrel{d}{=} \min _{1 \leq n \leq N}-\frac{I_{-n}}{n}-\max _{1 \leq n \leq N} \frac{I_{n}}{n}=\gamma_{0, N}^{-}-\gamma_{0, N}^{+} .
\end{aligned}
$$

Combining this with $\gamma_{k}^{-}-\gamma_{k}^{+} \geq \gamma_{k, N}^{-}-\gamma_{k, N}^{+}$shows (20). Applying the Markov Inequality, we see that

$$
\mathbb{E} \vartheta_{N} \geq 2 \mathbb{P}\left(\vartheta_{N} \geq 2\right)
$$


Using (16), (20), and (21), we thus obtain

$$
\begin{aligned}
c \ell(N)^{1 / 2} N^{H} & \geq \mathbb{E} F_{N}=\sum_{k=1}^{N-1} \mathbb{E}\left(\gamma_{k}^{-}-\gamma_{k}^{+}\right)_{+} \\
& \geq(N-1) \mathbb{E} \vartheta_{N} \geq 2(N-1) \mathbb{P}\left(\vartheta_{N} \geq 2\right) \\
& \geq 2(N-1) \mathbb{P}\left(\gamma_{0, N}^{-} \geq 1, \gamma_{0, N}^{+} \leq-1\right) \\
& =2(N-1) \mathbb{P}\left(I_{n}+|n| \leq 0:|n| \leq N\right) .
\end{aligned}
$$

Hence, we have for any $N$

$$
\mathbb{P}\left(I_{n}+|n| \leq 0:|n| \leq N\right) \leq c \ell(N)^{1 / 2} N^{-(1-H)} .
$$

\section{Lower bound for $\tilde{\boldsymbol{p}}_{N}$}

Along the lines of the proof of (20), one gets an analogous estimate when replacing $N$ by $\tilde{k}:=\min (k, N-k)$; namely

$$
\mathbb{E}\left(\gamma_{k}^{-}-\gamma_{k}^{+}\right)_{+} \leq \mathbb{E} \vartheta_{\tilde{k}} .
$$

Now, let $1-H<\alpha<1$. Then, we have by the monotonicity of $\vartheta_{N}$ for $N^{\alpha} \leq k \leq N-N^{\alpha}$

$$
\mathbb{E} \vartheta_{\tilde{k}} \leq \mathbb{E} \vartheta_{\left\lceil N^{\alpha}\right\rceil}
$$

Thus, by using (24) and (25), we obtain

$$
\begin{aligned}
\mathbb{E} F_{N} & =\sum_{k=1}^{N-1} \mathbb{E}\left(\gamma_{k}^{-}-\gamma_{k}^{+}\right)_{+} \\
& \leq\left(N-2\left\lfloor N^{\alpha}\right\rfloor\right) \mathbb{E} \vartheta_{\left\lceil N^{\alpha}\right\rceil}+2 \sum_{k=1}^{\left\lfloor N^{\alpha}\right\rfloor} \mathbb{E} \vartheta_{k} .
\end{aligned}
$$

Moreover, we know from (22), that we have for all $k$

$$
\mathbb{E} \vartheta_{k} \leq c \ell(k)^{1 / 2} k^{H-1} .
$$

Hence, by (26) and Proposition 1.5.8 in [6], we obtain

$$
\sum_{k=1}^{\left\lfloor N^{\alpha}\right\rfloor} \mathbb{E} \vartheta_{k} \leq c \ell\left(\left\lfloor N^{\alpha}\right\rfloor\right)^{1 / 2} N^{\alpha H} .
$$

Thus, we have

$$
c^{-1} \ell(N)^{1 / 2} N^{H} \leq \mathbb{E} F_{N} \leq N \mathbb{E} \vartheta_{\left\lceil N^{\alpha}\right\rceil}+c \ell\left(\left\lfloor N^{\alpha}\right\rfloor\right)^{1 / 2} N^{\alpha H} .
$$


Since $\alpha H<1$, we obtain

$$
c^{-1} \ell(N)^{1 / 2} N^{H-1} \leq \mathbb{E} \vartheta_{\left\lceil N^{\alpha}\right\rceil} .
$$

Replacing $N$ by $\left\lceil N^{1 / \alpha}\right\rceil$ yields

$$
\ell_{1}(N) N^{-(1-H) / \alpha} \leq \mathbb{E} \vartheta_{N},
$$

where $\ell_{1}$ is a slowly varying function at infinity.

Fix $q>1$ to be chosen later and let $\|\cdot\|_{q}$ denote the norm $\mathbb{E}\left[|X|^{q}\right]^{1 / q}$ for some random variable $X$. Then, using $\vartheta_{N} \leq \vartheta_{1}$ and Hölder's Inequality, we have

$$
\mathbb{E} \vartheta_{N}=\mathbb{E} \vartheta_{N} \mathbb{1}_{\vartheta_{N}>0} \leq \mathbb{E} \vartheta_{1} \mathbb{1}_{\vartheta_{N}>0} \leq\left\|\vartheta_{1}\right\|_{q} \mathbb{P}\left(\vartheta_{N}>0\right)^{1-1 / q}
$$

Further,

$$
\left\|\vartheta_{1}\right\|_{q} \leq\left\|I_{-1}-I_{1}\right\|_{q} \leq c \sqrt{q},
$$

using that $I_{-1}-I_{1}$ is a Gaussian random variable. So, we have

$$
\frac{\ell_{1}(N)}{c \sqrt{q}} N^{-(1-H) / \alpha} \leq \mathbb{P}\left(\vartheta_{N}>0\right)^{1-1 / q}
$$

Now, setting $q:=\log (N)+1$ yields

$$
\ell_{2}(N) N^{-(1-H) / \alpha} \leq \mathbb{P}\left(\vartheta_{N}>0\right)
$$

where $\ell_{2}$ is a slowly varying function at infinity.

In the following, we will relate the probability $\mathbb{P}\left(\vartheta_{N}>0\right)$ to the probability in (13). This is divided into four steps.

Step 1: We start with a change of measure argument. By Corollary 6 , we can find a function $f \in \mathcal{H}_{H}(I)$ such that $f(n) \geq \frac{3}{2}|n|$ for all $n \in \mathbb{Z}$. Then, using (6), we obtain

$$
\begin{aligned}
& e^{-\sqrt{2\|f\|^{2} \log \left(1 / \mathbb{P}\left(\vartheta_{N}>0\right)\right)}-\|f\|^{2} / 2} \mathbb{P}\left(\vartheta_{N}>0\right) \\
& \leq \mathbb{P}\left(\min _{-N \leq n \leq-1} \frac{I_{n}+f(n)}{n}-\max _{1 \leq n \leq N} \frac{I_{n}+f(n)}{n}>0\right) \\
& \leq \mathbb{P}\left(\min _{-N \leq n \leq-1} \frac{I_{n}+\frac{3}{2}|n|}{n}-\max _{1 \leq n \leq N} \frac{I_{n}+\frac{3}{2}|n|}{n}>0\right) \\
&=\mathbb{P}\left(\min _{-N \leq n \leq-1} \frac{I_{n}}{n}-\max _{1 \leq n \leq N} \frac{I_{n}}{n}>3\right) \\
&=\mathbb{P}\left(\vartheta_{N}>3\right) .
\end{aligned}
$$

So, by (27), $\mathbb{P}\left(\vartheta_{N}>0\right)$ and $\mathbb{P}\left(\vartheta_{N}>3\right)$ differ by less than a slowly varying function at infinity.

Step 2: Let

$$
A_{0}^{(N)}:=\left\{\left(x_{-N}, \ldots, x_{-1}, x_{1}, \ldots, x_{N}\right) \in \mathbb{R}^{2 N}: x_{n} \leq-|n|, 1 \leq|n| \leq N\right\},
$$


and let

$$
A_{m}^{(N)}:=A_{0}^{(N)}+m b^{(N)}, \quad \text { where } b^{(N)}:=(-N, \ldots,-1,1, \ldots, N) .
$$

In the following, we write $I \in A_{m}^{(N)}$ instead of $\left(I_{-N}, \ldots, I_{-1}, I_{1}, \ldots, I_{N}\right) \in$ $A_{m}^{(N)}$ for ease of notation. We will show that $\left\{\vartheta_{N}>3\right\} \subseteq \cup_{m \in \mathbb{Z}}\left\{I \in A_{m}^{(N)}\right\}$. For this purpose, let $m^{(N)}$ be an integer-valued random variable such that $\min _{-N \leq n \leq-1} \frac{I_{n}}{n} \in\left[m^{(N)}+1, m^{(N)}+2\right)$. Then, we obviously have $I_{n} \leq$ $\left(m^{(N)}+1\right) n$ for $-N \leq n \leq-1$. Furthermore, assuming $\vartheta_{N}>3$, we can conclude that

$$
\max _{1 \leq n \leq N} \frac{I_{n}}{n}<\min _{-N \leq n \leq-1} \frac{I_{n}}{n}-3<m^{(N)}-1 .
$$

Step 3: We show that $\mathbb{P}\left(I \in A_{0}^{(N)}\right) \geq \mathbb{P}\left(I \in A_{m}^{(N)}\right)$. For this purpose, we make use of an argument that is commonly used to prove Anderson's Inequality. It is well known that for any convex subsets $A, B \subseteq \mathbb{R}^{2 N}$ and $0<\lambda<1$, one has

$$
\mu(\lambda A+(1-\lambda) B) \geq \mu(A)^{\lambda} \mu(B)^{1-\lambda},
$$

where $\mu$ is a centered Gaussian measure on $\mathbb{R}^{2 N}$, see e.g. Theorem 2 in 15 . Since $\left(I_{-N}, \ldots, I_{-1}, I_{1}, \ldots, I_{N}\right)$ is a centered Gaussian random variable, by setting $\lambda=\frac{1}{2}$, we obtain

$$
\begin{aligned}
\mathbb{P}\left(I \in A_{0}^{(N)}\right) & =\mathbb{P}\left(I \in \frac{1}{2} A_{-m}^{(N)}+\frac{1}{2} A_{m}^{(N)}\right) \\
& \geq \mathbb{P}\left(I \in A_{-m}^{(N)}\right)^{1 / 2} \mathbb{P}\left(I \in A_{m}^{(N)}\right)^{1 / 2}=\mathbb{P}\left(I \in A_{m}^{(N)}\right) .
\end{aligned}
$$

Here, we used that one has $A_{0}^{(N)}=\frac{1}{2} A_{-m}^{(N)}+\frac{1}{2} A_{m}^{(N)}$ and, by symmetry of the process $\left(I_{n}\right), \mathbb{P}\left(I \in A_{-m}^{(N)}\right)=\mathbb{P}\left(I \in A_{m}^{(N)}\right)$.

Step 4: Now, we relate the quantities $\mathbb{P}\left(\vartheta_{N}>3\right)$ and $\tilde{p}_{N}$. Since $I_{-1}$ is a centered Gaussian random variable, we can choose a constant $c_{0}$ such that $\mathbb{P}\left(I_{-1} \leq-\left(a_{N}+1\right)\right) \in o\left(N^{-1}\right)$ for $a_{N}=\sqrt{c_{0} \log (N)}$. Further, by $\mathbb{P}\left(\cup_{m \geq a_{N}} A_{m}\right) \leq \mathbb{P}\left(I_{-1}<-\left(a_{N}+1\right)\right)$ and symmetry of the process $\left(I_{n}\right)$, we get

$$
\mathbb{P}\left(\cup_{|m| \geq a_{N}} A_{m}\right) \in o\left(N^{-1}\right) .
$$

Altogether we thus obtain

$$
\begin{aligned}
\mathbb{P}\left(\vartheta_{N}>3\right) & \leq \mathbb{P}\left(\cup_{m \in \mathbb{Z}} A_{m}\right) \\
& \leq \sum_{|m|<a_{N}} \mathbb{P}\left(A_{m}\right)+\mathbb{P}\left(\cup_{|m| \geq a_{N}} A_{m}\right) \\
& \leq 2 a_{N} \mathbb{P}\left(A_{0}\right)+2 \mathbb{P}\left(I_{-1} \leq-\left(a_{N}+1\right)\right) \\
& =2 a_{N} \mathbb{P}\left(I_{n}+|n| \leq 0:|n| \leq N\right)+o\left(N^{-1}\right) .
\end{aligned}
$$


Putting this together with (27) and (28), we get

$$
\ell_{3}(N) N^{-(1-H) / \alpha} \leq \mathbb{P}\left(I_{n}+|n| \leq 0:|n| \leq N\right),
$$

where $\ell_{3}$ denotes a slowly varying function at infinity.

\section{Polynomial rate of $\boldsymbol{p}_{N}$}

Clearly, we have from (29)

$$
\begin{aligned}
\ell_{3}(N) N^{-(1-H) / \alpha} & \leq \mathbb{P}\left(I_{n}+|n| \leq 0:|n| \leq N\right) \\
& \leq \mathbb{P}\left(I_{n} \leq 0:|n| \leq N\right)=p_{N} .
\end{aligned}
$$

In particular, $p_{N} \geq c^{-1} N^{-1}$ for some suitable constant $c$. This estimate will be used in the following change of measure argument. Due to Corollary 6 , we can choose a function $f \in \mathcal{H}_{H}(I)$ with $f(n) \geq|n|$ for all $n \in \mathbb{Z}$. Then, by (23) and Proposition 3, we obtain

$$
\begin{aligned}
c \ell(N)^{1 / 2} N^{-(1-H)} & \geq \mathbb{P}\left(I_{n}+|n| \leq 0:|n| \leq N\right) \\
& \geq \mathbb{P}\left(I_{n}+f(n) \leq 0:|n| \leq N\right) \\
& \geq \mathbb{P}\left(I_{n} \leq 0:|n| \leq N\right) e^{-\sqrt{2\|f\|^{2} \log \left(1 / p_{N}\right)}-\|f\|^{2} / 2} \\
& \geq \mathbb{P}\left(I_{n} \leq 0:|n| \leq N\right) e^{-\sqrt{2\|f\|^{2} \log (c N)}-\|f\|^{2} / 2} .
\end{aligned}
$$

Finally, we take log in (30), (31) and divide by $\log (N)$. Then, taking lim $\sup _{N}$ and liminf $\operatorname{in}_{N}$, respectively, and letting $\alpha \nearrow 1$ yields

$$
\lim _{N \rightarrow \infty} \frac{\log \left(\mathbb{P}\left(I_{n} \leq 0:|n| \leq N\right)\right)}{\log (N)}=H-1 .
$$

\section{References}

[1] Ash, R. B., And Gardner, M. F. Topics in Stochastic Processes, vol. 27 of Probability and Mathematical Statistics. Academic Press, New York, 1975.

[2] Aurzada, F., and Baumgarten, C. Persistence of fractional Brownian motion with moving boundaries and applications. Journal of Physics A: Mathematical and Theoretical 46, 12 (2013).

[3] Aurzada, F., And Dereich, S. Universality of the asymptotics of the one-sided exit problem for integrated processes. Annales de l'Institut Henri Poincaré Probabilités et Statistiques 49, 1 (2013), 236-251.

[4] Aurzada, F., Guillotin-Plantard, N., and PÃúne, F. Persistence probabilities for stationary increment processes. Stochastic Processes and their Applications, to appear, preprint available at arXiv:1606.00236. 
[5] Aurzada, F., And Simon, T. Persistence Probabilities and Exponents. In Lévy Matters $V$, vol. 2149 of Lecture Notes in Mathematics. Springer, Cham, 2015, pp. 183-224.

[6] Bingham, N. H., Goldie, C. M., and Teugels, J. L. Regular Variation. Encyclopedia of Mathematics and its Applications. Cambridge University Press, 1987.

[7] Bray, A. J., Majumdar, S. N., And Schehr, G. Persistence and First-Passage Properties in Nonequilibrium Systems. Advances in Physics 62, 3 (2013), 225-361.

[8] Castell, F., Gulllotin-Plantard, N., Pène, F., and Schapira, B. On the one-sided exit problem for stable processes in random scenery. Electronic Communications in Probability 18 (2013).

[9] Majumdar, S. N. Persistence in nonequilibrium systems. Current Science 77, 3 (1999), 370-375.

[10] Majumdar, S. N. Persistence of a particle in the Matheron-de Marsily velocity field. Physical Review E 68 (2003).

[11] Molchan, G. Maximum of a fractional Brownian motion: probabilities of small values. Communications in Mathematical Physics 205, 1 (1999), $97-111$.

[12] Molchan, G. The Inviscid Burgers Equation with Fractional Brownian Initial Data: The Dimension of Regular Lagrangian Points. Journal of Statistical Physics 167, 6 (2017), 1546-1554.

[13] Molchan, G., And Khokhlov, A. Small Values of the Maximum for the Integral of Fractional Brownian Motion. Journal of Statistical Physics 114, 3-4 (2004), 923-946.

[14] Oshanin, G., Rosso, A., And Schehr, G. Anomalous Fluctuations of Currents in Sinai-Type Random Chains with Strongly Correlated Disorder. Physical Review Letters 110 (2013).

[15] PrÉkopa, A. Logarithmic concave measures with application to stochastic programming. Acta Scientiarum Mathematicarum 32 (1971), 301-316.

[16] Redner, S. Survival probability in a random velocity field. Physical Review E 56 (1997), 4967-4972.

[17] Samorodnitsky, G. Long Range Dependence. Foundations and Trends® in Stochastic Systems 1, 3 (2006), 163-257. 
[18] She, Z.-S., Aurell, E., And Frisch, U. The Inviscid Burgers Equation with Initial Data of Brownian Type. Communications in Mathematical Physics 148, 3 (1992), 623-641.

[19] SinAi, Y. G. Statistics of Shocks in Solutions of Inviscid Burgers Equation. Communications in Mathematical Physics 148, 3 (1992), 601-621.

[20] Whitт, W. Stochastic-Process Limits. An Introduction to StochasticProcess Limits and Their Application to Queues. Springer Series in Operations Research and Financial Engineering. Springer, New York, 2002. 